\title{
CONSIDERATIONS IN FIRST-LINE THERAPIES FOR TREATMENT-NAÏVE CHRONIC HEPATITIS B
}

John Ryan, MD, Christian D. Stone, MD, Syed Abdul-Basit,MD, Karina Herrera, MD,. Department of Medicine at the University of Nevada School of Medicine

Address correspondence to:

John Ryan, MD

University of Nevada School of Medicine

2040 W Charleston Blvd. Suite 300

Las Vegas, NV 89102

jkryan@medicine.nevada.edu

\begin{abstract}
The treatment of chronic Hepatitis B Virus (CHBV) infection has evolved considerably during the last decade. As the number of potential therapies has grown, so has the relative complexity of managing the disease. There are now five oral nucleoside/nucleotide analogues and two injectable versions of interferon that are approved for the treatment of CHBV. Of these, the American Association for the Study of Liver Diseases (AASLD) now recommends only entecavir, tenofovir, and pegylated interferon as first-line options for treatment-naïve individuals. ${ }^{1,2}$ Each has some potential advantages and disadvantages that should be weighed prior to making a choice for what is generally long-term therapy.
\end{abstract}

Copyright (C) 2015, Knowledge Enterprises Incorporated. All rights reserved. 


\section{INTRODUCTION}

It is estimated that 350 million people worldwide are chronically infected with Hepatitis B Virus (HBV), and that within the US, at least 1.25 million are carriers of the disease. ${ }^{1-4}$ Between 15$40 \%$ of these carriers will go on to develop serious complications such as hepatic decompensation or hepatocellular carcinoma (HCC) ${ }^{5,6}$ There is data to support the benefits of therapy in preventing some of these sequelae in appropriate candidates.

Population studies indicate that the single best predictor of outcomes such as cirrhosis, HCC and mortality is HBV viral load. ${ }^{7}$ Incremental elevations in viral load seem to correlate with an increased risk of these complications. Inversely, viral load suppression has been associated with decreased ChildsPugh-Turcotte scores, improved fibrosis scores, cirrhosis regression, and decreased rates of HCC development. ${ }^{8-10}$ There is also data to indicate that the need for liver transplantation for decompensated HBV has decreased as a result of antiviral therapy. ${ }^{11}$

There are a number of predictors of treatment response such as viral load and liver enzyme elevations. ${ }^{1,2}$ Another emerging predictor is the viral genotype. There are eight HBV genotypes (A-H) described, each with a relatively characteristic geographic distribution. ${ }^{12}$ For instance, genotypes $\mathrm{A}$ and $\mathrm{D}$ are predominantly found in Europe, and B and $\mathrm{C}$ in Asia. ${ }^{12}$ The viral genotype may help to identify patients who are likelier to respond to antiviral therapy, as well as those who are more prone to developing the complications of their liver disease. $^{13-15}$
Patients at increased risk of hepatic complications should be considered for antiviral therapy. Pegylated interferon, entecavir and tenofovir are recommended as first-line options for treatment-naïve individuals. The choice of which agent to use depends on a number of factors including treatment tolerability, likelihood of response, and associated adverse effects. This review will highlight these issues to help tailor therapy to individual patients.

\section{PEGYLATED INTERFERON $\alpha 2 \mathrm{a}$}

Interferon is an immune modulator that is typically given for a finite duration of therapy. The use of pegylated interferon as an option to treat HBV has been somewhat limited in the US due to its side effect profile, and reduced efficacy in patients with genotype C. It has been used more extensively in Europe where favorable genotypes are more prevalent. It is probably most useful in patients who have high pre-treatment ALT, low viral load, and who have either genotype A or B virus. ${ }^{1,13-15}$

The original phase 3 registrational trials compared pegylated interferon to combination therapy with lamivudine and to lamivudine alone for 48 weeks. ${ }^{16}$, 17 Following the treatment period in studies in patients with hepatitis B e antigen $(\mathrm{HBeAg})$, the rate of viral suppression was highest in the combination therapy arm; however, eantigen seroconversion was the same for all groups. By the end of the 24-week follow-up period, these seroconversion rates had increased for the pegylated interferon (32\%), and combination (24\%) arms, whereas lamivudine remained significantly lower at $19 \%$. 
The combination arm was able to achieve the highest viral suppression rate following 48 weeks of therapy in patients without HBeAg. ${ }^{17}$ However, the defined endpoint of sustained response (viral suppression and biochemical response following a period of 26 weeks off-therapy) was similar for both interferon arms, and significantly better than for lamivudine alone.

There are reports of delayed s-Ag loss occurring in Caucasian patients treated with standard interferon. The rate of sAg loss has been noted to approach $65 \%$ in European studies, with much of this occurring following the treatment period. However, the phenomenon has not been seen in studies of Chinese patients. ${ }^{18-22}$

The major advantage of interferon-based therapy is the discrete treatment period, while the major disadvantage is its side effect profile. This includes hematologic effects, emotional lability, as well as fatigue, weight loss and hair loss. These side effects, as well as the potential for significant on-treatment transaminase flares, makes the drug particularly difficult to use in patients with decompensated liver disease.

Pegylated interferon $\alpha 2 \mathrm{a}$ is classified as category $\mathrm{C}$ for pregnancy. ${ }^{23}$ It should not be routinely used in this setting; however the discrete treatment course does make it an appealing choice for women of reproductive age with $\mathrm{CHB}$. There are no reported cases of HIVresistant mutations induced by interferon therapy. It is considered an appropriate monotherapy in HIV-infected individuals.

\section{ENTECAVIR}

Entecavir is a nucleoside analogue of guanosine. The original registrational trials compared entecavir $0.5 \mathrm{mg}$ to lamivudine $100 \mathrm{mg}$ for 48 weeks. ${ }^{24,25} \mathrm{At}$ the end of this period, in the e-antigen positive population, entecavir resulted in significantly higher rates of virologic (67\% vs. $36 \%)$, histologic $(72 \%$ vs. $62 \%$ ) and biochemical (68\% vs. 60\%) responses. The mean reduction in viral load with entecavir at this time point was $6.9 \operatorname{logs}$ (as compared to $5.4 \operatorname{logs}$ with lamivudine). A sub-group of patients from this study was followed for up to 5 years, demonstrating a viral suppression rate of $94 \%$ and a biochemical response rate of $80 \% .^{26}$ Of note, long-term intent-to-treat data is not available for this group of patients.

A second study comparing entecavir $0.5 \mathrm{mg}$ to adefovir $10 \mathrm{mg}$ daily again demonstrated its superiority in achieving rapid viral suppression (58\% vs. $19 \%$ at 12 weeks). ${ }^{27}$ As such, it appears to be a favorable choice of therapies for eantigen positive patients with high baseline viral loads.

For e-antigen negative patients, entecavir was again significantly better than lamivudine at achieving virologic $(90 \%$ vs. $72 \%)$, histologic (70\% vs. $62 \%)$ and biochemical (78\% vs. $71 \%)$ endpoints. $^{25}$ A sub-group of patients from this study was treated for 144 weeks, and was able to achieve a viral suppression rate of $95 \%$ and a biochemical response rate of $84 \% .^{28}$

There is only one randomized controlled trial directly comparing entecavir and tenofovir in patients with decompensated CHBV. Despite some limitations due to 
the small size of the study, at one year the efficacy of the two drugs appeared to be similar ${ }^{29}$.

The cumulative rate of resistance for entecavir in treatment naïve patients at six years has been reported as $1.2 \% .^{30} \mathrm{It}$ should be noted that the sixth year cohort represented only $15 \%$ of the original enrollment of the study, as patients who met certain pre-determined endpoints following the first and second years were removed from subsequent analyses by design. However, this low rate of resistance has been confirmed in real-world studies. ${ }^{31,32}$ It is postulated that the low incidence of long-term resistance can be largely attributed to the need for three individual point mutations in order to cause clinical resistance.

Entecavir appears to be a relatively safe drug. Early concerns regarding the potential for carcinogenicity have not materialized thus far. These concerns were based on data showing that lung adenomas could develop in mice exposed to the drug at 3-40 multiples of human dosing. ${ }^{33}$ A large post-marketing study mandated by the FDA is ongoing, however no evidence of increased carcinogenicity risk in humans has been demonstrated. ${ }^{26,34}$

There has also been a report on a series of five patients with advanced liver disease who developed lactic acidosis while being treated with entecavir. ${ }^{35}$ However, these patients had Model for End-stage Liver Disease (MELD) scores ranging from 22-38, and were at risk of developing lactic acidosis from other etiologies. Additionally, baseline lactic acid levels were not checked in this group. Finally, the time course from the start of the drug to the onset of lactic acidosis in at least three of the cases was likely too brief to be attributable to the drug. It should also be noted that mild increases in serum lactic acid levels have also been reported during the use of tenofovir monotherapy. ${ }^{36}$ As such, the evidence for an increased risk of lactic acidosis attributable specifically to entecavir use in patients with advanced liver disease is inadequate.

The experience with entecavir use during pregnancy is extremely limited. ${ }^{13}$ It is classified as pregnancy category $\mathrm{C}$ and should not be used routinely during pregnancy.

There have been reported cases of HIV resistance mutations occurring in coinfected patients treated with entecavir. ${ }^{1,33,37}$ Hence entecavir should not be used as monotherapy in the setting of HIV coinfection.

\section{TENOFOVIR}

Tenofovir is an oral acyclic nucleotide analogue of adenosine. It has been available for use in the treatment of HIV since 2001. It was additionally approved for the treatment of chronic HBV in 2008, and has since seen extensive use in this disease state. Clinical trials have demonstrated rates of virologic suppression of $93 \%$ and $76 \%$ at 48 weeks for e-Ag negative and e-Ag positive $\mathrm{CHBV}$ patients respectively. ${ }^{38}$ In both instances, the responses were significantly better than those observed with the comparison arm of adefovir. Additionally, in the e-Ag positive study, a rate of HBsAg loss of $3 \%$ was noted at the end of this period. Unfortunately, this endpoint was not achieved in any patients who had HBV genotypes B or C. 
Seven year data from the tenofovir HBV clinical trials has been reported. ${ }^{39,40}$ In an intention-to-treat analysis, the rates of virologic suppression at this time point were observed to be $77 \%$ for e-Ag negative patients, and $60 \%$ for e-Ag positive patients. . The rate of $\mathrm{HBsAg}$ loss at 7 years was reported as $12 \%$, but this continues to be limited to e-Ag positive patients who do not have genotypes B or C.

Histologic improvement at 5 years, defined as a Knodell inflamatory score improvement of at least 2 points without worsening of fibrosis, was seen in $88 \%$

No viral resistance to tenofovir has been reported to date in clinical trials. ${ }^{39,40}$ Of note, however, is that the study protocol allowed for patients with continued viremia at the 72 week time point to be given additional therapy with emtricitabine. This makes it difficult to assess resistance to tenofovir monotherapy beyond 72 weeks. However, from a practical standpoint, patients in clinical practice who continue to have elevated viral loads following 6 months of therapy should be offered alternative or additional treatment. ${ }^{1}$

Safety concerns regarding tenofovir have focused primarily on renal toxicity and bone density loss. Much of this discussion has occurred due to questions that have been raised by the HIV clinical experience. There have been specific reports of proximal tubular injury to the kidney, as well as Fanconi syndrome associated with tenofovir use. $^{41}$ Additionally, HIV clinical trials have raised the issue of bone density loss in patients on HAART with tenofovir. ${ }^{43}$
In HBV trials, as well as the subsequent open label phase, ten cases of confirmed increases in serum creatinine above $0.5 \mathrm{mg} / \mathrm{dL}$, and nine cases of hypophosphatemia have been observed over 336 weeks. Both of these numbers represent less than $1.5 \%$ of the original study cohort $(n=585)$. Additionally, there were 6 reports of confirmed decreases in creatinine clearance below the level of 50 during this period. ${ }^{39,40}$

The results of a partially matched retrospective analysis of 168 patients with $\mathrm{CHB}$, who were treated with either tenofovir or entecavir, support the clinical trial findings. In this analysis, confirmed increases in serum creatinine above $0.2 \mathrm{mg} / \mathrm{dL}$ were actually less common with tenofovir, however dose adjustments were more common with this drug. ${ }^{43}$

Four other clinical trials in various subpopulations have confirmed the general stability of serum creatinine and phosphate levels during TDF therapy. This raises the issue of whether following creatinine clearance is the best method of assessing for a proximal tubular injury. Tests such as serum creatinine and urine dipstick for albumin are better for detecting glomerular disease. $^{44}$ Proximal tubular dysfunction generally leads to wasting of substances that are filtered through the glomeruli and then reabsorbed at the level of the proximal tubule. Typical findings would hence be the presence of low molecular weight proteins, phosphorus and glucose in the urine. In its most severe form, this can manifest as Fanconi Syndrome with additional wasting of amino acids, uric acid and bicarbonate. Regular assessments of the protein:creatinine ratio of the urine, urinary retinol binding 
protein (RBP), as well as serum phosphate levels have been proposed as better tests of proximal tubular function (Table 1).

Data presented from a multicenter, cross-sectional study compared tubular fuction in patients exposed to a minimum of 2 years of tenofovir or emtricitabine. A significant higher rate of urine RBP excretion was found in the tenofovir treatment group. ${ }^{50}$

The same mechanism of proximal tubular dysfunction has been proposed as a possible etiology of bone mineral density loss with tenofovir use. ${ }^{44,45}$ It is thought that the resultant loss of phosphate, along with other minerals, may be at least partially responsible for this association. A further contribution to this process could result from altered gene expression in osteoblasts and osteoclasts due to preferential uptake of the drug. ${ }^{45}$

This concern for bone loss with tenofovir use stems largely from data derived from HIV clinical trials. These have demonstrated a statistically significant decrease in lumbar spine bone mineral density (BMD) during the first year of therapy on tenofovir-based HAART as compared to regimens without it. $^{42}$ It should be noted that following the initial year of therapy, this bone loss stabilizes, and it has not been associated with pathologic fractures. This has however, raised some concerns in the treatment of HBV, since Asian women are thought to have lower baseline BMD than Caucasians. $^{46}$ The manufacturers of tenofovir have added BMD assessments to their clinical trial protocols, so data on this topic should be forthcoming in the future. Unfortunately its value may be limited by the lack of baseline BMD measurements in these patients.

Tenofovir is categorized as pregnancy category B. Although experience with exposure to the drug during pregnancy is more extensive than with other first-line HBV therapies, the data is largely uncontrolled and selfreported. $^{47}$ Additionally there is the potential for fetal bone effects to be considered. $^{48}$ As such, routine therapy during early stages of pregnancy cannot be supported.

The use of tenofovir monotherapy in the setting of HIV is contraindicated due to the risk of inducing HIV resistance mutations. $^{41}$

Proposed labwork that may be useful to monitor during therapy with Tenofovir as well as the other first-line medications for chronic HBV is shown in table 1.

Table 1. Parameters to consider monitoring in patients on antiviral therapy ${ }^{\mathbf{4 4 , 4 5}}$

\begin{tabular}{|l|c|c|}
\hline \multicolumn{1}{|c|}{ All } & Pegylated Interferon & Tenofovir \\
\hline Liver panel & & $\begin{array}{c}\text { Urine Protein/Creatinine ratio } \\
\text { Viral load }\end{array}$ \\
$\begin{array}{l}\text { Electrolytes with anion gap } \\
\text { Renal function }\end{array}$ & CBC with differential & Bone scan \\
\hline
\end{tabular}




\section{CONCLUSIONS}

The current first-line therapies for HBV all appear to be relatively safe and effective. Since each has certain advantages, it is likely that as we learn more about therapy, it will be tailored to patient characteristics.

Interferon-based therapy may be preferred for young, female patients who are likely to become pregnant in the future. This may be particularly effective for Caucasian patients who have either genotypes A or B, or Asian patients with elevated transaminases. ${ }^{49}$ This group could then benefit from the advantage of a limited course of therapy, with some potential for inducing $\mathrm{HBsAg}$ seroconversion.

Entecavir may have some advantages for older patients at risk of osteopenia or renal dysfunction, who require treatment for CHBV. The safety, efficacy, and resistance profile, combined with current low cost of the medication, would also make entecavir a good choice for the general population, and specifically patients with high baseline viral loads.

Tenofovir has been used extensively in a young population with HIV. Therefore, it is likely also a good choice in young, otherwise healthy patients with $\mathrm{CHBV}$ (Figure 1). The acquisition of further data on the bone density effects of tenofovir in CHBV patients could clarify the role of the drug in populations at risk of osteopenia, such as the elderly. It has also been used extensively in combination with either lamivudine or emtricitabine. This experience is potentially useful for a population that is treatment-experienced, and in which the development of viral resistance could be a concern. Such patients may require many years of combination therapy.

Hence, all three first-line medications have desirable attributes for specific populations. As clinical experience and knowledge expand, it is probable that patient characteristics will guide therapeutic choices. 


\section{Figure 1. Algorithm for the treatment of chronic hepatitis B virus infection}

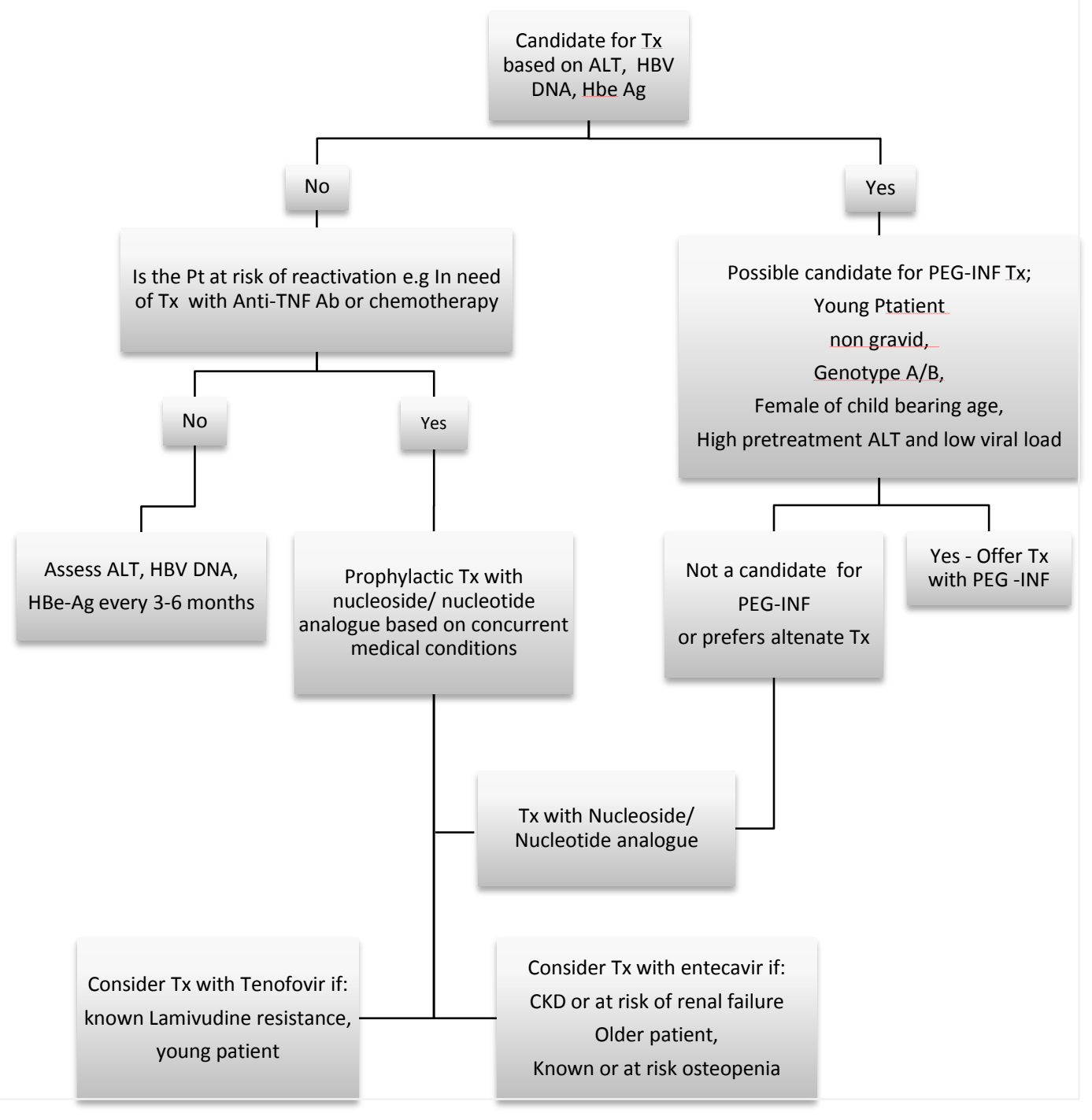

HBV-Hepatitis B virus, ALT- alanine transaminase, PEG-INF- pegylated interferon. 


\section{Conflict of Interest}

Guarantor of the article: John Ryan, MD. Specific Author Contributions: All authors were involved in the drafting and critical revision of the manuscript for important intellectual content.

Financial Support: None.

Potential Competing interests: the authors have nothing to disclose. 


\section{REFERENCES}

Lok ASF, Mcmahon BJ. Chronic hepatitis B. Hepatology 2009;50,No.3:1-36.

Keeffe E, Dieterich D, Han S, Jacobson I, Martin P, et al. An algorithm for the management of chronic hepatitis $B$ virus infection within the United States: 2008 Update. Clinical Gastroenterology and Hepatology; 6,12:1315-1341.

Lavanchy D. Hepatitis B virus epidemiology, disease burden, treatment and current and emerging prevention and control measures. J Viral Hepatitis 2004;11 (2):97-107.

Lee WM. Hepatitis B infection: New England Journal of Medicine 1997;337:1733-1745.

Bosch FX, Ribes J, Clerics R, Diaz M: Epidemiology of hepatocellular carcinoma: Clinics in Liver Disease 2005;9(2):191-211.

Beasley RP. Hepatitis B virus, the major etiology of hepatocellular carcinoma. Cancer 1988;61(10):1942-1956.

Chen CJ, Iloeje UH, Yang $\mathrm{HI}$ et al. Long-term outcomes in hepatitis B: The Reveal HBV Study; Clin Liv Dis. Nov 2007;797-816.

Liaw YF, Sung JJ, Chow WC et al. Lamivudine for patients with chronic HBV and advanced liver disease. N Eng J Med 2004;351(15):1521-1531.

Papatheodoridis GV, Lampertico P, Manolakopoulos $\mathrm{S}$ et al. Incidence of $\mathrm{HCC}$ in chronic HBV patients receiving nucleoside therapy: A systematic review.
Journal of Hepatology 2010; vol. 53:348356.

Chang TT, Liaw YF, Wu SS, Schiff E, Han KH, Lai CL, Safadi R, Lee SS, Halota W, Goodman Z, Chi YC, Zhang H, Hindes R, Iloeje U, Beebe S, Kreter B. Long-term entecavir therapy results in the reversal of fibrosis/cirrhosis and continued histological improvement in patients with chronic hepatitis B. Hepatology 2010; Sep;52(3):886-93

Kim WR, Benson JT, Hindman A, Brosgart C, Fortner-Burton C: Decline in the need for liver transplantation for end stage liver disease secondary to hepatitis B in the US. Hepatology 2007; Abstract 12.

Kidd-Lundggren K, Miyakawa Y, et al. Genetic Variability in hepatitis B virus. Journal of General Virology 2002;83:1267-1280.

Erhardt A, Heineke U, Blondin D et al. Mutations of the core promoter and response to interferon treatment in chronic replicative hepatitis B. Hepatology 2000;31:716-725.

Kao JK, Wu NH, Chen PJ et al. Hepatitis B genotypes and the response to interferon therapy. $\mathrm{J}$ Hepatology 2000;33:998-1002.

Wai CT, Chu CJ, Hussain M et al. HBV genotype $\mathrm{B}$ is associated with better response to interferon therapy in $\mathrm{HBe} A g$ positive chronic hepatitis than genotype C. Hepatology 2002;36:1425-1430.

Lau GK, Piratvisuth T, Luo KX et al. Peg Interferon alpha 2a, lamivudine, and the combination for $\mathrm{HBeAg}$-positive 
chronic HBV: New England Journal of Medicine 2005;352(26):2682-2695.

Marcellin P, Lau GK, Bowins $\mathrm{F}$ et al. Peg Interferon alpha 2a alone, lamivudine alone, and the two in combination in patients with $\mathrm{HBeAg}$ negative chronic HBV: New England Journal of Medicine 2004;351(12):12061217.

Niederau C, Heintges T, Lange $\mathrm{S}$ et al. Long-term follow-up of $\mathrm{HBeAg-positive}$ patients treated with interferon alfa for chronic hepatitis B. New Eng J of Med 1996;334(22):1422-1427.

Fattovich G, Giustina G, Realdi G et al. Long-term outcome of $\mathrm{HB}$ e-Ag-positive patients with compensated cirrhosis treated with interferon alfa (EUROHEP). Hepatology 1997;26(3):1338-1342.

Lin SM, Sheen IS, Chien RN et al. Long-term beneficial effect of interferon therapy in patients with chronic hepatitis B virus infection. Hepatology 1999;29(3):971-975.

Lau DT, Everhardt J, Kleiner DE et al. Long-term follow-up of patients with chronic hepatitis B virus infection. Gastroenterology 1997;113(5):16601667.

Lok ASF, Chung HT, Liu VW et al. Long-term follow-up chronic hepatitis B patients treated with interferon-alfa. Gastroenterology 1993;105(6):18331838.

The Antiviral Pregnancy Registry Interim Report; 1 January 1980 through 31 July 14, 2008.
Chang TT, Gish RG, de Man R et al. A Comparison of lamivudine and Entecavir for $\mathrm{HBeAg}$ positive chronic HBV. New England Journal of Medicine 2006;354(10):1001-1010.

Gish RG, Lok AS, Chang TT et al. Entecavir therapy for up to 96 weeks in patients with $\mathrm{HBeAg}$ positive chronic HBV. Gastroenterology 2007;133 (5):1437-1444.

Chang TT, Lai CL, Yoon SK et al. Entecavir treatment for up to 5 years in $\mathrm{HBeAg}$ positive chronic HBV. Hepatology 2010; 51:000-000.

Leung N, Peng CY, Hann HW et al. Early hepatitis B virus DNA reduction in hepatitis B e-antigen patients with chronic hepatitis B: A randomized international study of entecavir versus adefovir. Hepatology 2009; 49(1):72-79.

Shouval D. Three years of entecavir retreatment of $\mathrm{HBeAg}$ negative entecavir patients who previously discontinued entecavir treatment: Results from study Etv-901. AASLD 2008; Poster 893.

Liaw YF, Sheen IS, Lee CM, et al. Tenofovir disoproxil fumarate (TDF), emtricitabine/TDF, and entecavir in patients with decompensated chronic hepatitis B liver disease. Hepatology 2011; 53(1): 67-72.

Tenney DJ, Pokorowski KA, Rose RE et al. Entecavir maintains a high genetic barrier to $\mathrm{HBV}$ resistance through 6 years in naïve patients: EASL 2009; oral \#20.

Yuen MF, Seto WK, Fung J et al. Three years of continuous Entecavir therapy in treatment-naïve chronic $\mathrm{HBV}$ patients: 
viral suppression, viral resistance and clinical safety. APASL 2010; FP 95.

Lampertico P, Vigano M, Facheti F et al. Effectiveness of entecavir for nucleoside naïve $\mathrm{HBeAg}$-negative chronic hepatitis B patients in clinical practice: A 2-year multicenter cohort study in 311 patients. EASL 2010; poster 1009.

Baraclude Package Insert. Revised July 2009.

Manns M, Akarca US, Chang TT et al. Low rates of nucleoside-associated adverse events in the long-term experience with entecavir. EASL 2010; poster 1016.

Lange CM, Bojunga J, Hoffman WP. Severe lactic acidosis during treatment of chronic HBV with entecavir in patients with impaired liver function. Hepatology 2009;50(6):2001-2006.

Lange CM, Sprizl K, Veruchren J et al. "Prospective observation of lactate values during treatment of chronic Hepatitis B with nucleos(t)ide analogues in patients with decompensated liver cirrhosis" (Paper presented at the German Society of Internal Medicine Congress, Germany, 2010; PS 25.

Sasadeusz J, Audley J, Mijch A, Baden $\mathrm{R}$, Caro J, Hunter H, et al. The anti-HIV activity of entecavir: A multi-center evaluation of lamivudine-experienced and lamivudine-naïve patients. AIDS 2008;22:947-955.

Marcellin P, Heathcote EJ, Buti M, Gane E, De Man RA, et al. Tenofovir disoproxil fumarate vs. adefovir dipivoxil for chronic hepatitis B. N Eng J Med 2008;359:2442-2455.
Marcellin P, Gane E, Buti M, et al. Regression of cirrhosis during treatment with tenofovir disoproxil fumarate for chronic hepatitis B: a 5-year open-label follow-up study. Lancet. 2013 Feb 9;381(9865):468-75.

Marcellin P, Gane E, Tsai N, et al. "Seven years of treatment with tenofovir disoproxil fumarate for chronic hepatitis $B$ virus infection is safe and well tolerated and associated with Sustained virological, biochemical, and serological responses with no detectable resistance" (Paper presented at the 64rd Annual Meeting of the American Association for the Study of Liver Diseases Washington, DC Nov 1-5 2013).

Viread Package Insert. Revised March 2010.

Gallant J, Staszewski S, Pozniak AL et al. Efficacy and safety of tenofovir DF vs. stavudine in combination therapy in anti-retroviral-naïve patients: A 3-year randomized trial. 2004;(292)(2):191-201.

Gish RG, Clark MD, Kane SD, et al. Similar risk of renal events among patients treated with tenofovir or entecavir for chronic hepatitis B. Clinical Gastroenterology and Hepatoly. 2012 Aug; 10(8):941-946.

Woodward CLN, Hall AM, Williams AG et al. Tenofovir associated renal and bone toxicity. HIV Medicine 2009; 10:482-487.

Grigby IF, Pham L, Mansky LM et al. Tenofovir-associated bone density loss. Therapeutics and Clinical Risk Management 2010;6:41-47. 
Walker MD, Babbar R, Opotowsky AR et al. A referent BMD database for Chinese-American women. Osteoporosis International 2006;17:878887.

Squires K, Olmscheid B and S Zhang. Tenofovir-DF (TDF)-containing antiretroviral (ARV) regimens for treatment of HIV in pregnancy: Findings from the antiretroviral pregnancy registry (APR). 49th Interscience Conference on Antimicrobial Agents and Chemotherapy (ICAAC 2009). San Francisco. September 12-15, 2009; Abstract H-917.

Panel on treatment of HIV-infected women and prevention of perinatal transmission. Recommendations for the use of antiretroviral drugs in pregnant HIV-1 infected women for maternal health and interventions to reduce perinatal HIV transmission in the United States. May 24, 2010; pp 1-117.

Lok ASF, Lai CL, Wu PC et al. Longterm follow-up in a randomized controlled trial of recombinant alpha 2 interferon in Chinese patients with chronic HBV. Lancet 1988;2(8606):298302.

Rodriguez-Novoa S, Garcia-Samaniego J, Prieto M, Calleja JL, et al. Altered underlying tubular function in $\mathrm{HBV}$ monoinfected patients receiving nucleoside analogues in a real-world setting: preliminary findings of the MENTE study. AASLD 2013; Abstract 974. 